\title{
International Journal of Rheumatic Diseases
}

23rd Asia-Pacific League of Associations for Rheumatology (APLAR) Congress 28-31 August 2021

Kyoto International Convention Centre / streamed online

Publication of this supplement has been supported by Gilead Sciences K.K. 


\title{
International Journal of Rheumatic Diseases
}

\author{
Volume 24 | Supplement 2 | September 2021
}

\section{Contents}

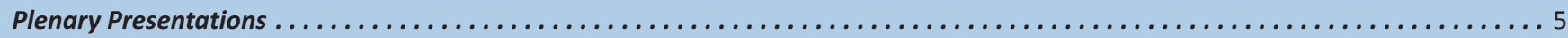

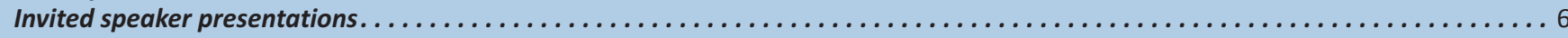

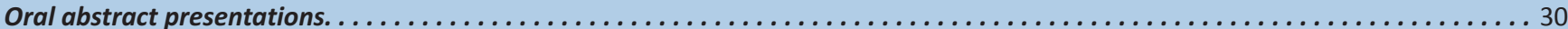

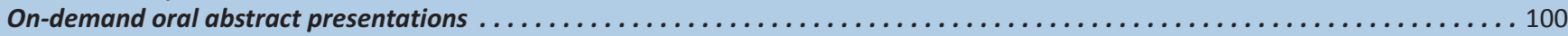

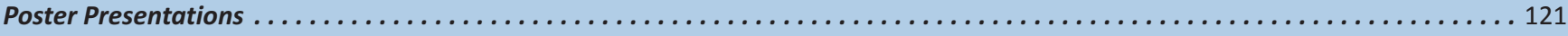

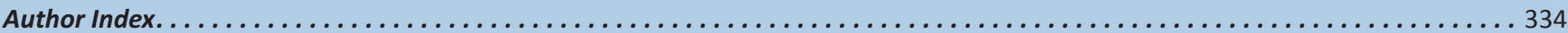


Distribution of Cases

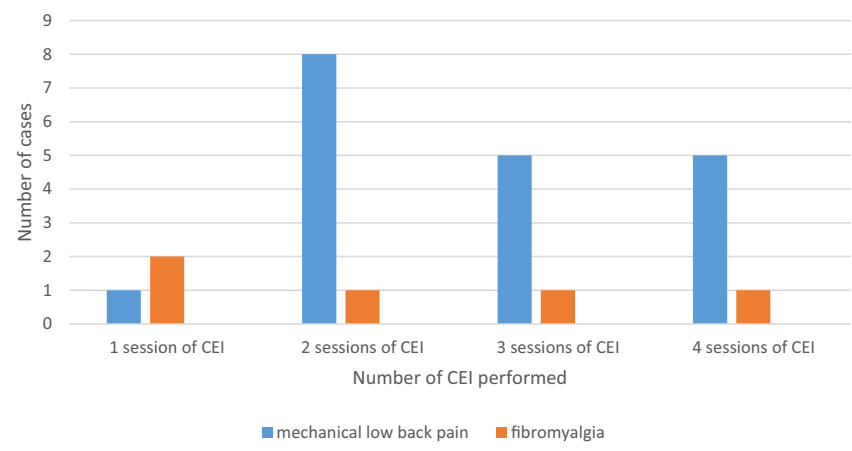

FIGURE 1 Distribution of cases according to number of CEI needed

fibromyalgia, remaining 5.7\% $(n=2)$ has underlying Polymyalgia rheumatica and psoriatic arthritis with axial involvement.

Baseline pain score was $6.7 \pm 1.6$, duration of CLBP was $3.0 \pm 2.9$ years. Average $3.6 \pm 2.1$ times of CEI were performed. $8.5 \%(n=3)$ had one CEI and remained pain free. $25.7 \%(n=9)$ had two CEI, $17 \%(n=6)$ three and another $17 \%(n=6)$ had four CEI as shown in Figure 1.

Pain score reduced to $2.9 \pm 1.3,1.9 \pm 1.4,1.4 \pm 1.4$ and subsequently $1.3 \pm 1.0$ following first, second, third and fourth CEI. On average PSR was $5.7 \pm 2.0$ ( $p=0.0005)$. This effect sustained for $6.3 \pm 4.3$ months. No complications were observed. $100 \%$ of them reported $50 \%$ or more PSR.

Conclusion: BD5\% in CEl helped to alleviate pain significantly. This is a good and safe alternative treatment in patients with CLBP.

\section{Enzymatic activities of the purine metabolism enzymes in lyzed erythrocytes of rheumatoid arthritis patients during NSAIDs treatment}

M. Mamus $^{1}$, E. Mozgovaya ${ }^{1}$, S. Bedina ${ }^{1}$, S. Spitsina ${ }^{1}$, A. Trofimenko ${ }^{1}$ ${ }^{1}$ Research Institute of Clinical and Experimental Rheumatology named after A.B. Zborovsky, Volgograd, Russian Federation

Objective: Evaluation of adenosine deaminase (ADA), purine nucleotide phosphorylase (PNP), xanthine oxidase (XO), and xanthine dehydrogenase $(X D H)$ in lyzed erythrocytes obtained from RA patients during NSAIDs treatment.

Methods: The research was conducted in accordance with the Declaration of Helsinki. 46 RA patients conformed to EULAR/ACR diagnostic criteria (2010) with moderate disease activity by DAS28 index were included in the research. 35 healthy subjects were enrolled as a reference group for calculation of reference ranges of the enzymatic activities $(M \pm 3 S D)$. RA patients were randomized into two comparable groups, first one treated with diclofenac sodium (Diclofenac, Hemofarm) 75 mg daily, and the second one treated with ketoprofen (Ketonal, Sandoz Novartis) 100 mg daily. Paired blood samples were obtained before and 30-35 min after the first NSAID injection. Erythrocytes were purified by means of the density gradient centrifugation. Lysis of red blood cells was induced by triple freezing-thawing. Enzymatic activities were measured spectrophotometrically, normalized to $10^{9}$ cells, activity changes are expressed as increase rates \pm SEM. The differences are considered significant when $\mathrm{p}<0.05$.

Results: Both groups of RA patiens had high pre-treatment activities of ADA ( $p<0.01)$, XO ( $p<0.001)$, PNP $(p<0.001)$, and low XDH activity $(p<0.001)$ in lyzed erythrocytes. There was substantial decrease of PNP activity after diclofenac injection (-3.37 $\pm 0.23 \%$; $p<0.001$ ), while 3 other enzymatic activities revealed no significant changes. In contrast with diclofenac, there were marked decrease of $\mathrm{XO}, \mathrm{PNP}$, and ADA activities in erythrocytes after ketoprofen injection $(-5.31 \pm 0.26 \%, p<0.01 ;-2.88 \pm 0.26 \%, p<0.01 ;-3.0 \pm 0.2 \%$; $p>0.05$, respectively). On the contrary, $\mathrm{XDH}$ activity after ketoprofen injection was somewhat higher $(4.69 \pm 0.18 \% ; p<0.001)$.

Conclusion: Single intramuscular injection of diclofenac or ketoprofen produced therefore unidirectional action on enzymatic activities studied. Decrease of XO activity in lyzed erythrocytes was more prominent after ketoprofen, and decrease of PNP activity - after diclofenac treatment. Both NSAIDs can influence on all the enzymatic activities, promoting their return into normal ranges.

\section{Allied Health: Alternative Medicine}

\section{Complimentary alternative medicine use among rheumatology patients: a comparative study between India and UK Rheumatology Teaching Hospitals}

S. Sai GopalaKrishnan ${ }^{1}$, J. Visalakshi Srikanth ${ }^{1}$, C. Tharakan ${ }^{2}$, J. Sabu $^{2}$, A. Moorthy ${ }^{2}$

${ }^{1}$ Amrita Vishwa Vidyapeetham, Cochin, India; ${ }^{2}$ University Hospitals of Leicester NHS trust, Leicester, UK

Background: Complementary and alternative medicines (CAM) are widely used by Rheumatology patients. We attempted to evaluate CAM usage among two different health system and health belief in the world and compare patient reported usage of CAM in Southern part of India and a UK teaching hospital.

Methods: This is an observational, semiqualitative study using piloted questionnaires in English which was distributed to rheumatology outpatient services, in India and UK. 250 successive patients from India completed the questionnaires, which was compared with 107 of UK Respondents. The data was analysed using excel spreadsheet and SPS version 20. Local ethics committee approved this study.

Results: Total respondents 357, $n=357$.The gender is equally distributed with mean age of 44 years. Ethinicity includes both Asian and White with Asian as predominant. Rheumatoid arthritis and fibromyalgia patients are frequent users of CAM. 33.2\% of the Indian patients and $31.8 \%$ of UK patients reported using CAM. The frequency of CAM usage varies with $13.1 \%$ using CAM daily. Ayurveda 